\title{
Low-dimensional dynamical systems for a wake-type shear flow with vortex dislocations
}

\author{
Hongliang Zhao ${ }^{\mathrm{a}, \mathrm{b}, *}$, Guocan Ling ${ }^{\mathrm{a}}$ \\ ${ }^{a}$ State Key Laboratory of Nonlinear Mechanics, Institute of Mechanics, Chinese Academy of Sciences, \\ Beijing 100080, China \\ ${ }^{\mathrm{b}}$ Research Center for Fluid Dynamics, College of Science, PLA University of Science and Technology, \\ Nanjing 211101, China
}

Received 17 October 2002; received in revised form 24 June 2003; accepted 24 June 2003

Communicated by M. Yamada

\begin{abstract}
Low-dimensional systems are constructed to investigate dynamics of vortex dislocations in a wake-type shear flow. High-resolution direct numerical simulations are employed to obtain flow snapshots from which the most energetic modes are extracted using proper orthogonal decomposition (POD). The first 10 modes are classified into two groups. One represents the general characteristics of two-dimensional wake-type shear flow, and the other is related to the three-dimensional properties or non-uniform characteristics along the span. Vortex dislocations are generated by these two kinds of coherent structures. The results from the first 20 three-dimensional POD modes show that the low-dimensional systems have captured the basic properties of the wake-type shear flow with vortex dislocation, such as two incommensurable frequencies and their beat frequency.
\end{abstract}

(c) 2003 Published by The Japan Society of Fluid Mechanics and Elsevier B.V. All rights reserved.

PACS: 47.20.Ky; 47.27.Vf; 47.27.Cn

Keywords: POD; Navier-Stokes equations; Vortex dislocations

\section{Introduction}

A number of experimental studies, such as Williamson (1992), Dallard and Browand (1993), Lewis and Gharib (1992) among others, have shown that in the cylinder wake, mixing layer and some other nonlinear waves the flow transition involves the appearance of large-scale vortex dislocations or a

\footnotetext{
* Corresponding author.

E-mail address: zhao_hongliang@hotmail.com (H. Zhao).
} 
pattern 'defect' with a complex three-dimensional configuration. Vortex dislocations are generated between spanwise vortex shedding cells out of phase. They play an important role in the flow transition and are considered to be a new kind of the mechanism for the transition. Recently, Ling and Xiong (2001) and Braza et al. (2001) have made numerical exploration on the vortex dislocations in a wake-type flow and a cylinder wake. The generation of forced vortex dislocations and natural vortex dislocations and their influences on the flow were reported. Some classical fluid dynamics research methods and local analysis techniques such as wavelet analysis have been used to study vortex dislocations. However, there has been almost no report using proper orthogonal decomposition (POD) methods to describe the vortex dislocations or constructing low-dimensional dynamical systems using POD to study the dynamical properties of the vortex dislocations.

The forced vortex dislocations are sometimes well-organized large-scale flow structure and appeared periodically. The POD is a rational decomposition technique in which the most energetic modes may be extracted systematically from an ensemble of signals provided by a set of snapshots of flow field from experiments or numerical simulations. The modes can be used as a basis for a Galerkin reduction of the governing equations. Background material for the POD approach can be found in the review article by Berkooz et al. (1993). Recently, a number of fluid mechanical problems, including the near-wall boundary layer (e.g., Aubry et al., 1988; Rempfer, 1996) and the channel flow (Moin and Moser, 1989; Bonnet et al., 1994; Bangia et al., 1997 and others) have been studied using POD method.

This study tries to simplify the complex vortex shedding phenomena induced by a cylinder with spanwise diameter variation, using a wake-type shear flow profile with local spanwise variation in streamwise velocity defect, and to gain some basic knowledge on the mechanism of vortex dislocations appearing in the real flows. Following the 'concept of computational reducibility' by Triantafyllou and Karniadakis (1990), it is expected that the vortex wake will be reproduced numerically by only knowing the time-averaged velocity profile at specific locations behind a circular cylinder, where it is most unstable. According to the linear instability analysis with different time-averaged streamwise inlet velocity profiles obtained from previous experiments, we can get vortex streets with different shedding frequencies along the span of the flow. By studying the evolution of this kind of flow, we expect to obtain the vortex dislocation phenomena with saving large computation resources. It is also suggested that the results from this study will not be the same as those given by the experiments, but they may mimic, in some basic aspects, the vortex dislocations produced by a real cylinder with disturbance or a variation in cylinder diameter. It is presumptive that the present results will be helpful to understand the complexity on the vortex dislocation occurring in real cylinder wakes. On the other hand, because the evolution of the wake-type shear flow leads to periodic vortex shedding, it also can be considered as a kind of the nonlinear waves. Thus, the present work also relates to the vortex dislocation problem in nonlinear waves. Direct numerical simulations of the vortex dislocations in a flow past a real cylinder and physical analysis are now undertaken, and will be reported in a succeeding paper.

In the present work, some basic features of the vortex dislocations in the wake-type shear flow are numerically simulated. The three-dimensional characteristics of the flow are investigated by POD method. Properties of the low-dimensional dynamical systems with vortex dislocations using POD are discussed. This paper is organized as follows. In Section 2, we briefly present the spectral direct numerical simulation (DNS) to obtain spatio-temporal evolution of the wake-type shear flow with local spanwise non-uniformity. In Section 3, we briefly describe the POD procedure and the resulting 
POD vector modes for the wake-type shear flow. In Section 4, the low-dimensional dynamical systems are constructed based on the varying number of these eigenmodes, $N=10-30$, using linear Galerkin projections, and we conclude the paper with a discussion in Section 5.

\section{Numerical simulations}

\subsection{Theoretical formulation and numerical method}

In order to obtain high numerical accuracy and, wavenumber resolution a compact finite differenceFourier spectral hybrid method is used for solving three-dimensional Navier-Stokes equations. A detailed presentation of these equations and the numerical method can be found in Xiong and Ling (1996). The procedure of the method is summarized as follows. In the spanwise direction of the flow, the periodic boundary conditions are assumed, and then we expand all the flow variables into a truncated Fourier series as

$$
\varphi(x, y, z, t)=\sum_{m=-N / 2}^{N / 2-1} \varphi_{m}(x, y, t) \mathrm{e}^{-\mathrm{i} m \beta z},
$$

where $x, y$ and $z$ are the streamwise, vertical, and spanwise directions, respectively. $N$ is the cutoff, and $\beta$ is the spanwise wavenumber. The three-dimensional incompressible Navier-Stokes equations are written, in primitive variable formulation, as

$$
\frac{\partial \boldsymbol{V}}{\partial t}+(\boldsymbol{V} \cdot \nabla) \boldsymbol{V}=-\nabla p+\frac{1}{R e} \nabla^{2} \boldsymbol{V}
$$

where $\boldsymbol{V}=\{u, v, w\}$ are the velocity components in $x, y, z$ directions, respectively, and $p$ is the pressure. The characteristic length $D$ and the velocity scales $U_{0}$ are introduced, where $U_{0}$ is specified to be the streamwise wake-type inflow velocity $U(y, z)$ at $y= \pm \infty$, and $D$ is chosen to be the half-width of the wake-type inflow so that, in non-dimensional variables, $U(D / 2, \infty)=\frac{1}{2}[U(0, \infty)+$ $U(\infty, \infty)]$. The Reynolds number is defined as $R e=U_{0} D / v$, where $v$ is the kinematic viscosity.

Substituting (2.1) into (2.2), we find a system of equations for the $m$ th harmonic in a twodimensional $(x, y)$ domain as

$$
\frac{\partial \boldsymbol{V}_{m}}{\partial t}+F_{m}[(\boldsymbol{V} \cdot \nabla) \boldsymbol{V}]=-\nabla_{m} p_{m}+\frac{1}{R e} \nabla_{m}^{2} \boldsymbol{V}_{m}
$$

where $\nabla_{m} \equiv\{\partial / \partial x, \partial / \partial y,-\mathrm{i} m \beta\}, \nabla_{m}^{2} \equiv \partial^{2} / \partial x^{2}+\partial^{2} / \partial y^{2}-m^{2} \beta^{2}$, and $F_{m}[(\boldsymbol{V} \cdot \nabla) \boldsymbol{V}]$ is the Fourier transformation of the nonlinear terms. For the time discretization of the equation a third-order mixed explicit-implicit schemes is used. The solution procedure at every time step is split into three substeps. The pseudo-spectral method is adopted to evaluate the nonlinear terms in the split equations. When the Fourier coefficients of velocities are transformed into the velocities in the physical space, the nonlinear convection terms are approximated by the fifth-order upwind compact scheme. For solving the Helmholtz equations for pressure and velocity, a nine-point compact scheme of fourth-order accuracy is derived and the fourth-order central compact scheme is used for the non-homogeneous term. In this method a semi-discrete pressure boundary conditions and a generalized non-reflecting outlet boundary conditions are used. 


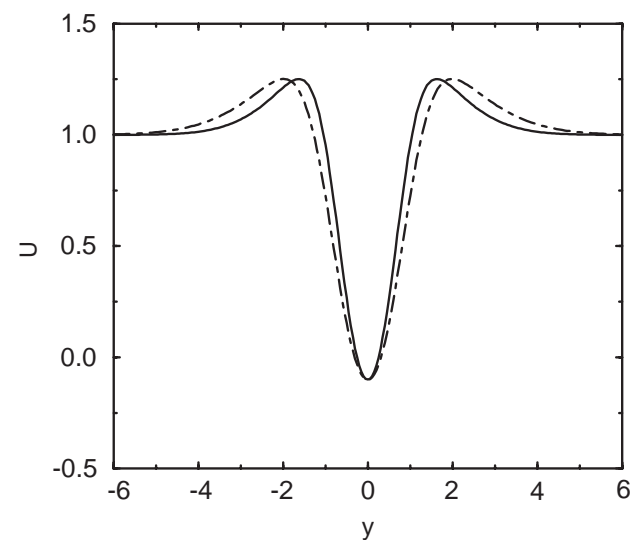

Fig. 1. Inflow velocity profile $U(y, z)$ at different span locations, where solid line is for $2.5<|z|<15$ and dot-dashed line is for $|z| \leqslant 2.5$.

The wake-type inflow velocity profile is taken as

$$
U(y, z)=1.0-a(z)\left(2.0-\cosh (b(z) y)^{2}\right) \mathrm{e}^{-(c(z) \cdot y)^{2}},
$$

which represents the time-averaged streamwise velocity profile in wake near the cylinder near wake where the flow is most unstable as seen in the direct numerical simulations of Karniadakis and Triantafyllou (1992) as well as the experimental measurement of Nishioka and Sato (1974).

Here, $a(z), b(z)$, and $c(z)$ are the parameters imposed to produce different inlet velocity profiles along the span of the flow. In the present calculation, $a(z)=1.1, b(z)=1.1, c(z)=1.2$ for the outer region $|z|>2.5$, and $a(z)=1.1, b(z)=0.9, c(z)=0.9818$ for the central non-uniform region $|z| \leqslant 2.5$. These variations of the parameters yield two different inlet velocity profiles along the span. It may mimic, to some extent in some basic aspects, the situation of cylinder with stepped variation of the spanwise diameter. The inlet velocity profiles at different regions are shown in Fig. 1, where $D=1.42$, $U_{0}=1$ and $R e=200$. The computation domain is 60,30 , and 30 in the streamwise, vertical, and spanwise directions, respectively. The cutoff wavenumber of the truncated Fourier series is $N=64$, and the corresponding number of the grid points in $(x, y)$ plane is $122 \times 62$.

\subsection{General feature of vortex dislocations}

A typical signal of the vertical velocity component $v$ is presented in Fig. 2. The sampling points are taken on a line of $x=15$ and $y=0.5$ for all $z$ positions examined. The vertical velocity component is employed for the analysis, because it responds immediately to the passage of a von Kármán vortex row and it is not masked by the upstream convection velocity as in the case of the streamwise component. The signal has normal characteristics of two dimensional wake flow at $z \geqslant 5$, while the signal is modified at $z \in(0,3)$, and is qualitatively similar to those with vortex dislocations observed in experiments, such as Williamson (1992), and in numerical simulations, such as Braza et al. (2001).

In order to examine the global behavior of the flow along the spanwise direction, a fast Fourier transformation was performed on the $v$ component signals at different values of $z$, each of which 


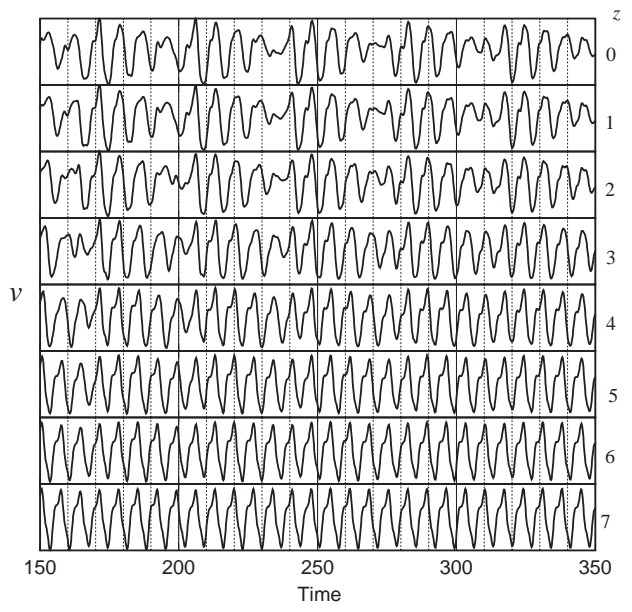

Fig. 2. Time series of vertical velocity component $v$ at different positions $z$ along the span at $(x, y)=(15,0.5)$.

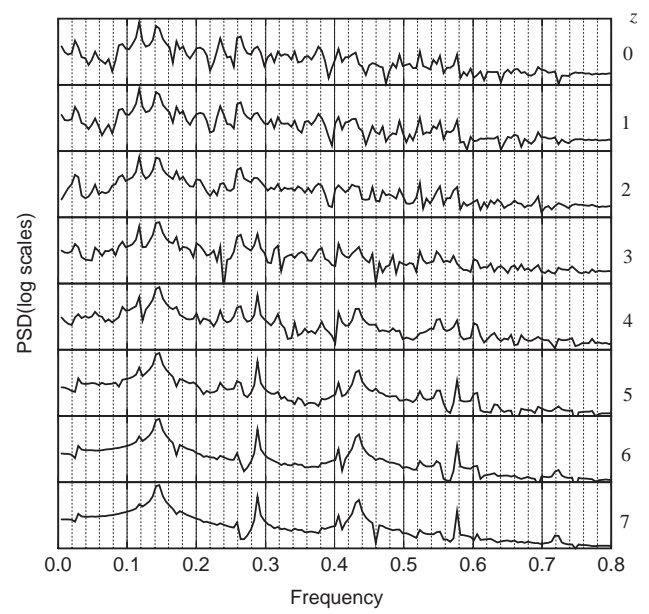

Fig. 3. Power Spectral Density (PSD) of vertical velocity component $v$ at different positions $z$ along the span at point $(x, y)=(15,0.5)$.

consists of 1024 points. The dimensionless length of the time series was 0.2 and the dimensionless frequency accuracy was 0.0049 . The spectra of vertical velocity component $v$ for several values of $z$ are shown in Fig. 3, where we can see two incommensurable frequencies, i.e. $f_{1}=0.141$, $f_{2}=0.117$, from $z=0$ to 3 , while there only appears a single peak $f_{1}=0.141$ at $z \geqslant 4$. The two incommensurable frequencies, and the relative energies of the two peaks, are primarily functions of the spanwise location. Furthermore, for $z \in(0,3)$, a peak appears in the spectrum at a reduced frequency of 0.0244 , i.e. at the difference frequency between the two peaks. It seems that this apparent 'beat' frequency, $f_{1}-f_{2}$, is the result of nonlinear interaction of the two shedding frequencies of neighboring cells. 

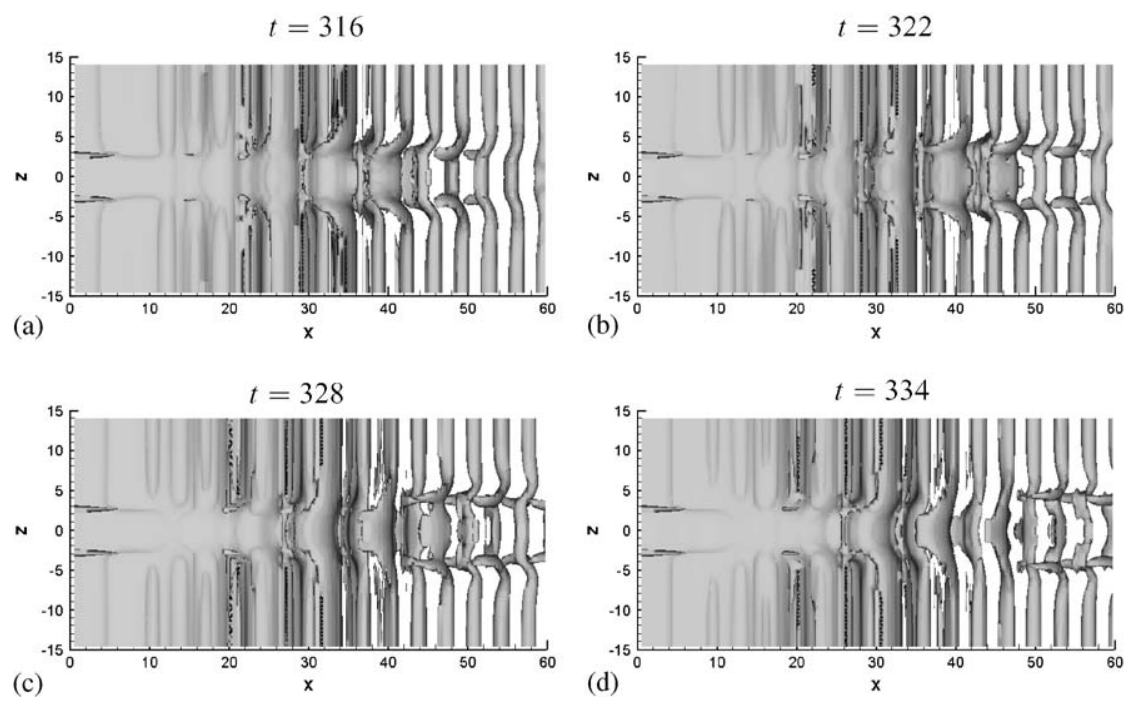

Fig. 4. Iso-vorticity surfaces at values $|\omega|=0.15$.
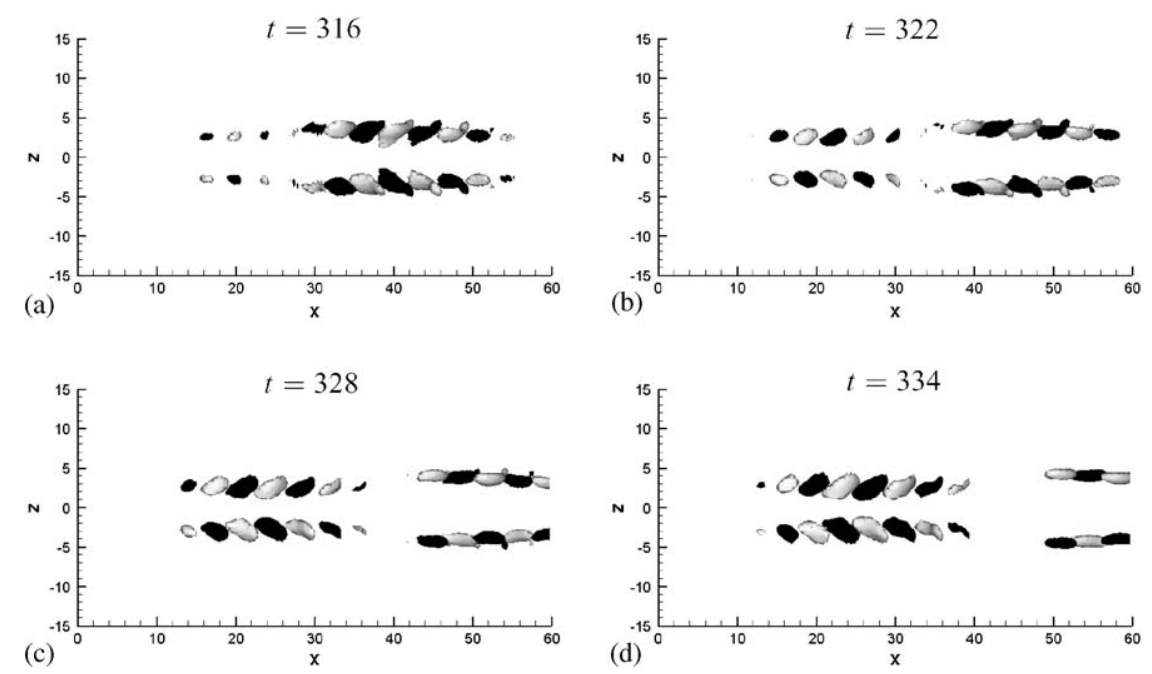

Fig. 5. Iso surfaces at values $\omega_{x}=0.11$ (gray) and -0.11 (black).

Iso-surfaces of vorticity and iso-surfaces of the streamwise vorticity component are shown, respectively, in Figs. 4 and 5, which show the formation of vortex dislocation in this flow. In Fig. 4, the spanwise vortex rolls are largely modified along the span. Streamwise and vertical vorticity branches are deformed at the position of large curved vortex rolls and connected with adjacent vortices with opposite sign. The vortex linkage region is in a narrow zone near the central area. In Fig. 5, the iso-vorticity surface of $\omega_{x}= \pm 0.11$ shows the structure of the linkage. It is shown that the vortex dislocations do not occur uniformly in the streamwise direction. The flow pattern is a 
chain-like structure in this direction. The head part (downstream) of the structure is narrow, the width of which is about 5. The bottom (upstream) of it is wider, and is about 10 in width. A gap appears between these two adjacent structures. As seen in the sequential figures from (a) to (d), the chain-like structure in the downstream moves out of the computation region and at the same time a new vortex dislocation structure is generated in the upstream. In the downstream near the positions $|z|=2.5$, the flow pattern is very like the direct mode in Lewis and Gharib (1992). As the central nonuniform region is not very long in the spanwise direction, the pattern may be concerned as a spot-like structure which is similar to the two-side vortex dislocations in Williamson (1992).

This is the general feature of vortex dislocations in the wake-type shear flow. More detailed features and a case of different discontinuous inlet profile will be discussed in our succeeding work. In the next section, we will focus on the wake-type shear flow by analyzing the POD eigenmodes and a low dimensional dynamical system derived by using the eigenmodes.

\section{Eigenspectrum and POD modes}

In order to obtain the most energetic eigenmodes from the DNS databases we employ the snapshot method of Sirovich (1987), which we formulate as follows.

Let $\boldsymbol{V}\left(\boldsymbol{x}, t_{m}\right)$ be a time-dependent velocity field, where $t_{m}$ for $m \in[1, M]$ is a sequence of discrete time at which the snapshots are taken, and $M$ is the total number. We then decompose $\boldsymbol{V}\left(\boldsymbol{x}, t_{m}\right)$ as

$$
\boldsymbol{V}\left(\boldsymbol{x}, t_{m}\right)=\boldsymbol{U}(\boldsymbol{x})+\boldsymbol{u}\left(\boldsymbol{x}, t_{m}\right),
$$

where $\boldsymbol{U}(\boldsymbol{x})$ is the average and $\boldsymbol{u}$ defines the fluctuation velocity, and its covariance matrix is obtained by

$$
C_{p, q}=\int_{\Omega} \boldsymbol{u}\left(\boldsymbol{x}, t_{p}\right) \cdot \boldsymbol{u}\left(\boldsymbol{x}, t_{q}\right) \mathrm{d} \Omega,
$$

where $\Omega$ is the computational domain. Let $\left(b_{k}^{1}, \ldots, b_{k}^{M}\right)^{\mathrm{T}}$ denotes the eigenvector to $C_{p, q}$ with corresponding eigenvalue $\lambda_{k}(1 \leqslant k \leqslant M)$. The sum of these eigenvalues gives the total fluctuation velocity 'energy' $E$ of the system

$$
E=\sum_{k=1}^{M} \lambda_{k},
$$

where the snapshots number $M \geqslant n^{*}$ is determined by the numerical cutoff criterion $\lambda_{n^{*}} / \lambda_{1}=10^{-8}$. The POD vector mode $\phi_{k}(\boldsymbol{x})$ is defined by

$$
\boldsymbol{\phi}_{k}(\boldsymbol{x})=\frac{\sum_{m=1}^{M} b_{k}^{m} \boldsymbol{u}\left(\boldsymbol{x}, t_{m}\right)}{\left\|\sum_{m=1}^{M} b_{k}^{m} \boldsymbol{u}\left(\boldsymbol{x}, t_{m}\right)\right\|} .
$$

This equation is written in matrix form, and the eigenspectrum and corresponding eigenmodes are obtained using standard LINPACK routines. The normalized POD modes thus obtained are numerically orthogonal. 


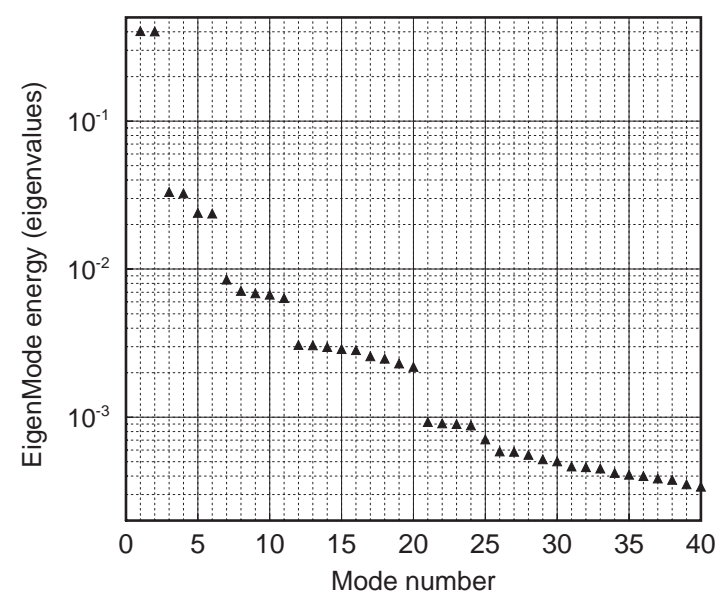

Fig. 6. Eigenvalues normalized by the total energy of the fluctuation velocity system for three-dimensional wake-type shear flow at $R e=200$.

The eigenvectors are, by construction, incompressible,

$$
\nabla \cdot \boldsymbol{\phi}_{k}(\boldsymbol{x})=0 \quad(k=1, \ldots, M) .
$$

The fluctuation velocity at any instant can be expanded in terms of these POD vector modes as

$$
\boldsymbol{u}\left(\boldsymbol{x}, t_{m}\right)=\sum_{k=1}^{M} a_{k}\left(t_{m}\right) \boldsymbol{\phi}_{k}(\boldsymbol{x}),
$$

where $a_{k}\left(t_{m}\right)$ is the amplitude.

We perform the decomposition using 160 snapshots from our simulation ensemble. In Fig. 6 we show the first 20 largest eigenvalues corresponding to the most energetic modes. The first 10 modes are found to occur in pairs of eigenvalues of comparable magnitude. The modes form pairs due to the closeness of the vortex street to a traveling wave, similarly to the two-dimensional flow studied in Deane et al. (1991). This is also reflected in the eigenmodes of each pair, which are only phase-shifted with respect to each other. But after the 10th mode, the modes do not appear in pairs. The first two modes dominate over the fluid motion, comprising more than $80 \%$ of the total fluctuation velocity energy of the motion. The first 20 modes collectively contain more than $98 \%$ of the energy.

In analyzing the modes, it should be kept in mind that each POD mode does not necessarily represent a coherent structure, while the sum of several modes most probably does. Each mode can be thought to capture dominant characteristics of the flow which may or may not render itself to visualization or other diagnostic techniques.

A qualitative picture of the structure of the POD modes is provided in Fig. 7 which presents the first through 10th modes of the vertical component of eigenmodes. In this figure, each one in the first five pairs of modes presents almost the same pattern but with phase shift each other. These are three-dimensional global eigenmodes, for which it becomes ambiguous how scale information is introduced as the mode index increases. The first, the third and the fifth pairs of modes still show that the structures are refined as the mode index increases. These three pairs of modes 

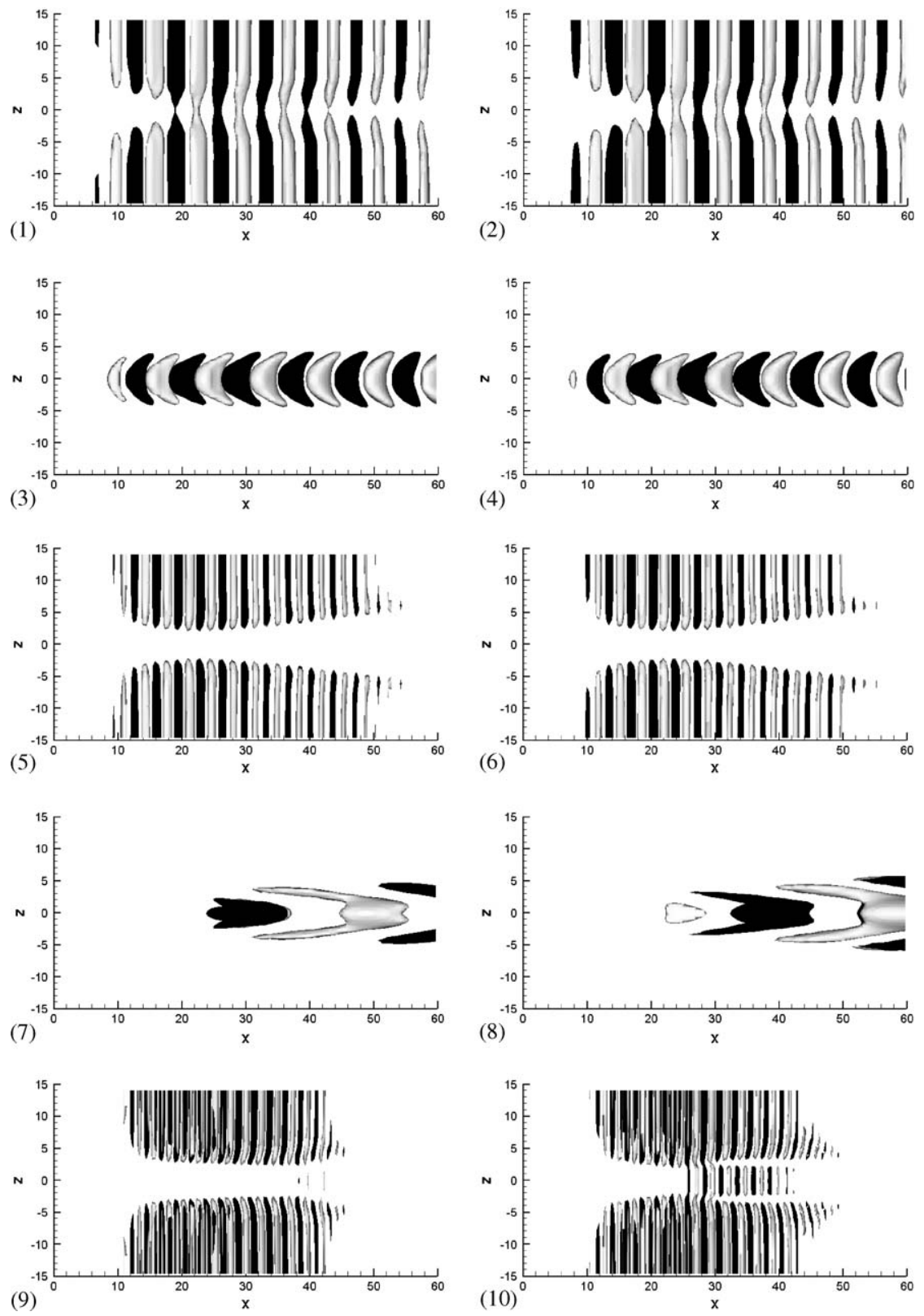

Fig. 7. Contours of the vertical component of the first 10 modes at values 0.01 (gray) and -0.01 (black).

also show two-dimensional characteristics except that there is a break at the middle region of the spanwise.

An interesting thing is that the second and the fourth pairs of modes are concentrated in the middle region of the span where the other three pairs of modes are not large. The size of the two pairs of modes in the streamwise direction is larger than the other three pairs in the first five pairs 

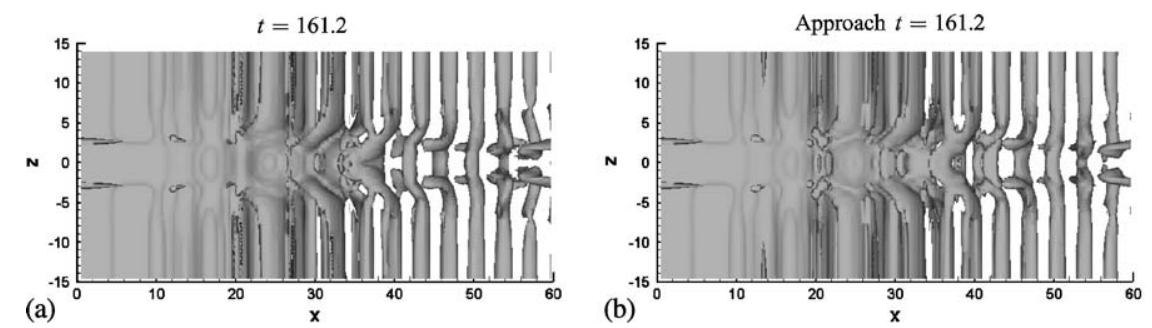

Fig. 8. Iso-vorticity surfaces at values $|\omega|=0.15$ : (a) DNS, and (b) reconstructed result by the first 20 POD modes.

of modes. More interesting is that the size of the fourth pair modes is the largest one in the first five pairs. In $z$ direction, the width of the structure in the upstream is much larger than that in the downstream. So we may say that the second pair of modes mainly corresponds to the characteristics of the non-uniform region, and the fourth pair of modes primarily represents the characteristics of the vortex dislocations.

Finally, we note that based on the energy decay shown in the eigenspectrum plot in Fig. 6, it appears that at least the first 20 modes are energetically significant and should be included in the truncated system. Actually the first 20 modes are used to reconstruct the flow (Fig. 8(b)) almost the same as the result of the direct simulation (Fig. 8(a)).

\section{Construction of dynamical systems}

The dynamical systems are obtained by performing a Galerkin projection of the incompressible Navier-Stokes equations onto the space of the eigenmodes. Expanding the velocity as in (3.1) we form the evolution equations for the amplitudes in (3.6) through

$$
\int_{\Omega} \boldsymbol{\phi}_{k}(\boldsymbol{x}) \cdot\left(\frac{\partial \boldsymbol{u}}{\partial t}+(\boldsymbol{V} \cdot \nabla) \boldsymbol{V}+\nabla p-\frac{1}{R e} \nabla^{2} \boldsymbol{V}\right) \mathrm{d} \Omega=0
$$

and

$$
\int_{\Omega} \phi_{k}(\boldsymbol{x})(\nabla \cdot \boldsymbol{V}) \mathrm{d} \Omega=0 .
$$

The divergence-free equation (4.2) is satisfied automatically since the POD modes are divergence-free (3.5) by construction. The pressure term drops out from the governing equations since

$$
\int_{\Omega} \boldsymbol{\phi}_{k}(\boldsymbol{x}) \cdot \nabla p \mathrm{~d} \Omega=\int_{\Gamma} p \boldsymbol{\phi}_{k} \cdot \boldsymbol{n} \mathrm{d} \Gamma-\int_{\Omega}\left(\nabla \cdot \boldsymbol{\phi}_{k}\right) p \mathrm{~d} \Omega=0,
$$

where $\Gamma$ is the boundary of $\Omega$, and $\boldsymbol{n}$ is its unit normal outward vector. The boundary conditions on the flow are periodic in the spanwise direction. Furthermore, the pressure is zero at the outflow boundary, while the eigenvector are zero at the inflow boundary by construction. Hence, there is no contribution from the first term in (4.3). 

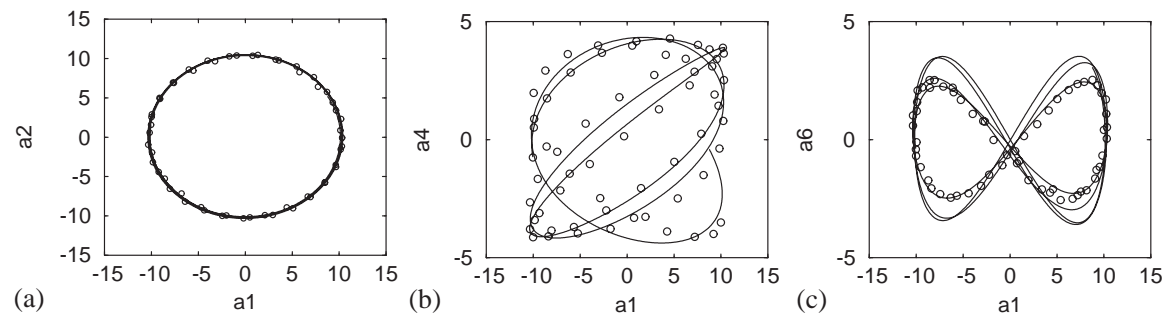

Fig. 9. Phase portraits of the coefficients. The circles correspond to the coefficients which are directly obtained by projection of the DNS data on POD modes, and the curves to the coefficients of POD prediction.

The above procedure leads to a system of $N$ equations by retaining the first $N$ modes from $M$ POD modes in the Galerkin expansion

$$
\frac{\mathrm{d} a_{k}}{\mathrm{~d} t}=-\sum_{l} \sum_{m} B_{k l m} a_{l} a_{m}-\sum_{l} C_{k l} a_{l}-D_{k},
$$

where

$$
\begin{aligned}
B_{k l m} & =\int_{\Omega}\left(\boldsymbol{\phi}_{l} \cdot \nabla \boldsymbol{\phi}_{m}\right) \cdot \boldsymbol{\phi}_{k} \mathrm{~d} \Omega, \\
C_{k l} & =\int_{\Omega}\left(\boldsymbol{U} \cdot \nabla \boldsymbol{\phi}_{l}\right) \cdot \boldsymbol{\phi}_{k} \mathrm{~d} \Omega+\int_{\Omega}\left(\boldsymbol{\phi}_{l} \cdot \nabla \boldsymbol{U}\right) \cdot \boldsymbol{\phi}_{k} \mathrm{~d} \Omega-\frac{1}{R e} \int_{\Omega} \nabla^{2} \boldsymbol{\phi}_{l} \cdot \boldsymbol{\phi}_{k} \mathrm{~d} \Omega, \\
D_{k} & =\int_{\Omega}(\boldsymbol{U} \cdot \nabla \boldsymbol{U}) \cdot \boldsymbol{\phi}_{k} \mathrm{~d} \Omega-\frac{1}{R e} \int_{\Omega} \nabla^{2} \boldsymbol{U} \cdot \boldsymbol{\phi}_{k} \mathrm{~d} \Omega .
\end{aligned}
$$

In (4.4), the constant terms arise from the contribution of the mean flow, the linear terms involve the time-varying part (including its interaction with the mean), and the quadratic terms are due to the self-interaction of the time-varying part.

We have obtained the low-dimensional systems for $N=10,20$, and 30. The system with 10 modes displays an oscillation which, however, slowly grows without bound. But using 20 and 30 modes, the oscillations are stabilized. The simulation with $N=30$ modes is almost indistinguishable from the simulation with $N=20$ modes for the history of the coefficients of the first 10 modes.

A comparison of the time coefficients of the model system with $N=20$ modes to those of the full system is shown in Figs. 9 and 10 for 400 convective time units (only parts of them are plotted in the figures) at $R e=200$. The limit cycle is reasonably accurately captured by the first and second modes (Fig. 9(a)). The shape of the attractor in the phase portrait of $a-a_{6}$ is almost the same as that of $a_{1}-a_{3}$ in the two-dimensional case in Deane et al. (1991), while the shape of $a_{1}-a_{4}$ is new, which means there are new characteristics in the system for the three-dimensional properties of the flow.

It is shown in Fig. 10 that the amplitude of the time coefficients decreases as the index increases. The coefficients of the first four modes in the low-dimensional dynamical system match well with the coefficients which are directly obtained by projection of the DNS data on POD modes. However for the higher modes, e.g., the fifth and the sixth modes in the figure, there are noticeable differences of the amplitudes while the phases still match very well, though for the seventh and the eighth modes 


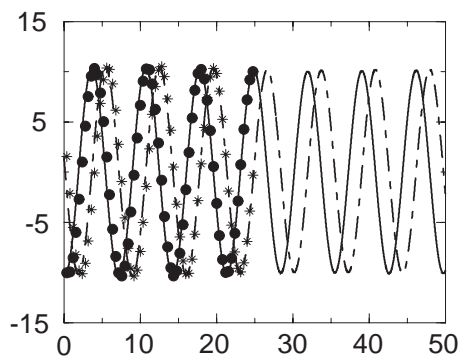

(a)

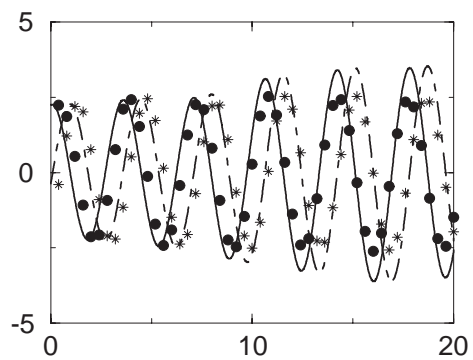

(c)

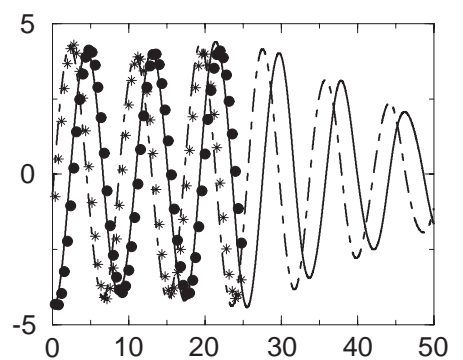

(b)

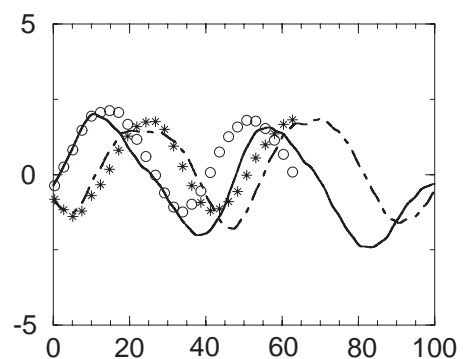

(d)

Fig. 10. Time history of the coefficients of different modes of the low-dimensional system with 20 POD modes: (a) $a_{1}, a_{2}$, (b) $a_{3}, a_{4}$, (c) $a_{5}, a_{6}$, and (d) $a_{7}, a_{8}$. The dots and stars correspond to the coefficients which are directly obtained by projection of the DNS data on POD modes, and the solid curves and dashed curves to the coefficients of POD prediction.
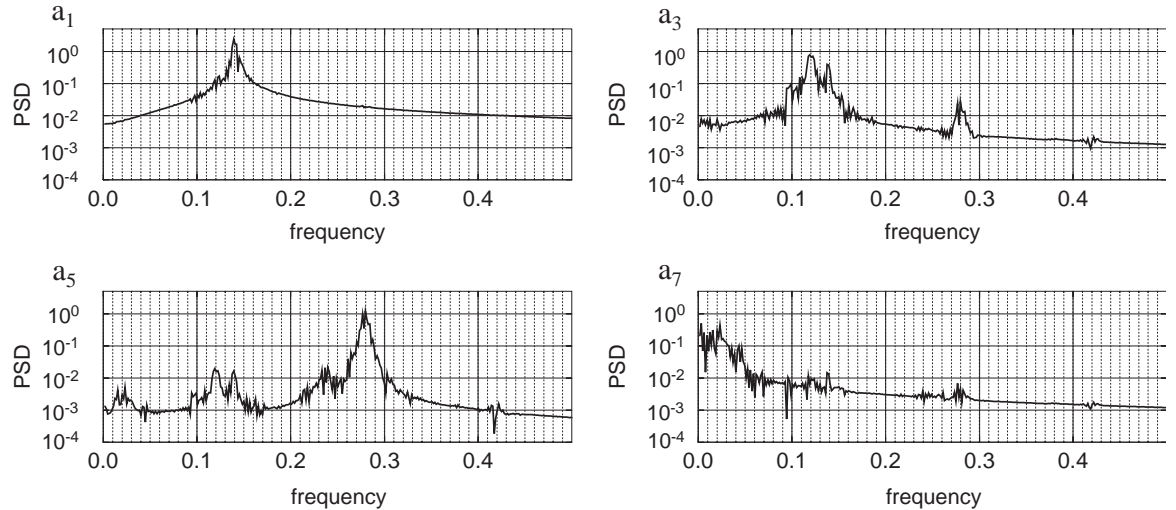

Fig. 11. Spectra of the coefficients $a_{1}, a_{3}, a_{5}$, and $a_{7}$.

the coefficients could match only qualitatively. Comparing the time history of coefficients of the first four pairs of modes, we find that the amplitude and the period of each pair are the same except for the phase shift each other.

The coefficients of the first eight modes are chosen for the fast Fourier transform analysis. The properties of the odd and the even modes of each pair are the same and the results of odd modes are drawn in Fig. 11. The amplitude $a_{1}$ has the frequency $f_{1}^{\prime}=0.140$, which is almost equal to $f_{1}$ 
in the DNS data analysis. The frequency spectrum of $a_{5}$ has a peak at twice of $f_{1}^{\prime}$. The spectrum of $a_{3}$ has the frequency $f_{2}^{\prime}=0.117$, which is equal to $f_{2}$ in the DNS data analysis. The beat frequency, $f_{1}-f_{2}$, in the DNS frequency analysis appears in the spectrum of $a_{7}$.

In summary, the low-dimensional system of $N=20$ not only captures well the basic characteristics of the real flow, but also clearly presents the difference of dynamical roles of the coefficients. The amplitudes $a_{1}$ and $a_{5}$ correspond to the characteristics of the basic flow, $a_{3}$ has a property of the non-uniform region and $a_{7}$ represents the dynamical features of the vortex dislocations.

\section{Conclusion and discussion}

The POD procedure has been used to obtain low-dimensional systems. It is found that 20 POD modes are sufficient to reproduce the three-dimensional wake-type shear flow with vortex dislocations. The characteristics of the first 10 modes and their dynamical properties are studied in detail.

The POD modes have captured the coherent structure of the three-dimensional wake-type shear flow. There are two kinds of POD modes. One kind of modes, such as the first, the third and the fifth pair modes, represent the general characteristics of two-dimensional basic flows, while the other one, including the second and the fourth pair of modes, is mainly related to the three-dimensional properties or non-uniform characteristics in the spanwise direction; the second pair of modes represents the characteristics of the non-uniform region of the wake-type flow, and the fourth pair of modes represents the characteristics of the vortex dislocations.

The orbit of the low-dimensional dynamical system, which we are concerned with, is stable, and lasts for a few hundred shedding cycles. The base frequency $\left(f_{1}\right.$, one of the two incommensurable frequencies) and its second and third harmonics are, respectively, described by the coefficients of the first, the third and the fifth pair of modes in the phase space. The spectral properties of the flow, such as $f_{2}$ (the other one of the two incommensurable frequencies) and the beat frequency, are also accurately described in the dynamical system by the coefficients of the second and the fourth pair of modes.

The present study has shown that the POD method can well extract the coherent structures of wake-type shear flows with vortex dislocation, the low-dimensional dynamical system constructed by using the POD modes can clearly reproduce the dynamical properties of the flow; the fourth pair of modes represents the characteristics of the vortex dislocations and their coefficients show the beat frequency, $f_{1}-f_{2}$. In physical space, these properties correspond to the vortex dislocations in the wake-type shear flow.

The flow field and the vortex dislocations obtained in the present work are specified by the inlet flow with stepped variation in velocity defect. When the width between the discontinuity is decreased as $a(z)=1.1+0.4 \mathrm{e}^{-z^{2}}$ (Ling and Xiong, 2001), a systematic vortex dislocation is also generated, while the flow pattern and the frequency variation in downstream are not the same as the flow presented here. Various vortex dislocation modes and three-dimensionality could be produced. However, features on the generation of vortex dislocations are similar; for instance, streamwise vorticity is generated or split from the original spanwise rolls and connected to adjacent spanwise vortex rolls by substantial redistribution of the vorticity. Details will be reported in later works. 


\section{Acknowledgements}

The authors would like to cordially acknowledge Prof. M. Yamada and Prof. S. Kida for their valuable suggestions and helping to improve the manuscript. This work is supported by the Special Funds of Major State Basic Research Projects (G1999032801) and National Natural Science Foundation of China (10272104). Computational resources are provided by LSSC computer center, CAS.

\section{References}

Aubry, N., Holmes, P., Stone, E., Lumley, J.L., 1988. The dynamics of coherent structures in the wall region of a turbulent boundary layer. J. Fluid Mech. 192, 115-173.

Bangia, A.K., Batcho, P.F., Kevrekidis, I.G., Karniadakis, G.E.M., 1997. Unsteady two-dimensional flows in complex geometries: comparative bifurcation studies with global eigenfunction expansions. SIAM J. Sci. Comput. 18, $775-805$.

Berkooz, G., Holmes, P., Lumley, J.L., 1993. The proper orthogonal decomposition in the analysis of turbulent flows. Ann. Rev. Fluid Mech. 25, 539-575.

Bonnet, J.P., Cole, D.R., Delville, J., Glauser, M.N., Ukeiley, L.S., 1994. Stochastic estimation and proper orthogonal decomposition: complementary techniques for identifying structure. Exp. Fluids 17, 307-314.

Braza, M., Faghani, D., Persillon, H., 2001. Successive stages and the role of natural vortex dislocation in three-dimensional wake transition. J. Fluid Mech. 439, 1-41.

Dallard, T., Browand, F.K., 1993. The growth of large scales at defect sites in the plane mixing layer. J. Fluid Mech. 247, 339-368.

Deane, A.E., Kevrekidis, I.G., Karniadakis, G.E., Orszag, S.A., 1991. Low dimensional models for complex geometry flows: application to grooved channels and circular cylinders. Phys. Fluids A 3, 2337-2354.

Karniadakis, G.E., Triantafyllou, G.S., 1992. Three-dimensional dynamics and transition to turbulence in the wake of bluff objects. J. Fluid Mech. 238, 1-30.

Lewis, C.G., Gharib, M., 1992. An exploration of the wake three dimensionalities caused by a local discontinuity in cylinder diameter. Phys. Fluids A 4 (1), 104-117.

Ling, G.C., Xiong, Z.M., 2001. Generation of large-scale vortex dislocations in a three-dimensional wake-type flow. Sci. China Ser. A 44 (12), 1585-1595.

Moin, P., Moser, R.D., 1989. Characteristic-eddy decomposition of turbulence in a channel. J. Fluid Mech. 200 , $471-509$.

Nishioka, M., Sato, H., 1974. Measurements of velocity distributions in the wake of a circular cylinder at low Reynolds numbers. J. Fluid Mech. 65, 97-112.

Rempfer, D., 1996. Investigations of boundary layer transition via Galerkin projections on empirical eigenfunctions. Phys. Fluids 8, 175-188.

Sirovich, L., 1987. Turbulence and the dynamics of coherent structures, Parts I, II and III. Q. Appl. Math. XLV, $561-590$.

Triantafyllou, G.S., Karniadakis, G.E., 1990. Computational reducibility of unsteady viscous flow. Phys. Fluids A 2, $653-658$.

Williamson, C.H.K., 1992. The natural and forced formation of spot-like vortex dislocations in the transition of a wake. J. Fluid Mech. 243, 393-441.

Xiong, Z.M., Ling, G.C., 1996. Compact finite difference-Fourier spectral method for three-dimensional incompressible Navier-Stokes equation. Acta Mech. Sinica 12 (4), 296-306. 